\title{
Optimal design of supply chain network under uncertainty environment using hybrid analytical and simulation modeling approach
}

\author{
N. Chiadamrong ${ }^{1} \cdot$ V. Piyathanavong ${ }^{1}$
}

Received: 6 July 2016/ Accepted: 10 April 2017/Published online: 17 April 2017

(C) The Author(s) 2017. This article is an open access publication

\begin{abstract}
Models that aim to optimize the design of supply chain networks have gained more interest in the supply chain literature. Mixed-integer linear programming and discrete-event simulation are widely used for such an optimization problem. We present a hybrid approach to support decisions for supply chain network design using a combination of analytical and discrete-event simulation models. The proposed approach is based on iterative procedures until the difference between subsequent solutions satisfies the pre-determined termination criteria. The effectiveness of proposed approach is illustrated by an example, which shows closer to optimal results with much faster solving time than the results obtained from the conventional simulation-based optimization model. The efficacy of this proposed hybrid approach is promising and can be applied as a powerful tool in designing a real supply chain network. It also provides the possibility to model and solve more realistic problems, which incorporate dynamism and uncertainty.
\end{abstract}

Keywords Hybrid optimization - Supply chain network design - MILP · Simulation-based optimization · Uncertainty

N. Chiadamrong

navee@siit.tu.ac.th

1 School of Manufacturing Systems and Mechanical Engineering, Sirindhorn International Institute of Technology, Thammasat University, Pathumthani 12121, Thailand

\section{Introduction}

A supply chain is a network of participants (e.g., suppliers, manufacturers, warehouses, distributors, and retailers) who, through coordinated plans and activities, develop products by converting raw materials into finished products (Chandra and Grabis 2007; Izadi and Kimiagari 2014). While companies rush where they want to compete in their industry's value chain, they are cooperating more and more in their business activities with external participants. Whether to establish a new supply chain or reconfigure an existing supply chain can be one of the major decisions to be made. This problem is a critical issue in achieving supply chain improvement as it can guarantee the success of the entire network performance (Tabrizi and Razmi 2013). Therefore, supply chain optimizations are subject to a particularly comprehensive evaluation, which, in turn, requires utilization of a variety of models and tools.

Supply chain optimization involves making decisions for planning and design of production, storage locations, flow of quantity among facilities, and transportation in the chain, which are essential for retaining the competitive edge of companies in a global economy (Mehrdad et al. 2015). These problems are often very large and complex due to the large number of facilities of the supply chain, such as the number of plants, warehouses, and retailers, and due to complex interactions among these facilities, such as the modes of transport, relocation of warehouses, and the stochastic nature of demand, etc. (Nikolopoulou and Ierapetritou 2012; Tavakkoli-Moghaddam et al. 2013). Moreover, analytical modeling somehow provides exact and static information, which is uncertain for real-world problems. The complexity of such problems causes huge computational load and time, which is beyond the ability of 
pure analytical modeling. As a result, analytical models often require many simplifying assumptions.

As computer technology and simulation software have advanced, the cost of computer time has become much cheaper, and simulation software has become more widely available. Use of simulation models for complex systems has become more feasible and popular. This simulation tool is used to capture the behavior of all the entities involved, their interactions, and the uncertainties associated with these systems. The choice between simulation and analytical models now depends primarily on the nature of the application, required accuracy, and availability of solution procedures. Making more use of one or the other during the various stages of problem solving can result in tremendous cost savings. Banks et al. (2001) noted the advantages and disadvantages of using analytical and/or simulation models.

Hybrid analytical and simulation modeling is a suitable framework that can incorporate the uncertainties in the stochastic supply chain network design problem. We compare the proposed hybrid approach with the traditional analytical model and simulation-based optimization model to understand their differences along with their advantages and disadvantages in a practical decision making context by presenting our findings with computational analysis. This hybrid approach combines both analytical and simulation modeling based on the development of independent mathematical and simulation models of the supply chain network, which are combined together for problem solving. The coupling of the agent-based model built for simulating the supply chain network with the optimization tool is developed using an iterative process. To demonstrate this approach, a profit maximization problem of the production-distributionstorage of a supply chain network is used as a test example, and the results are discussed. The rest of this paper is organized as follows. In Sect. "Literature review", a review of related literature is provided. Section "Supply chain network design" discusses the structure and operations of a supply chain network. In Sect. "Hybrid analytical-simulation modeling approach", the proposed hybrid analytical and simulation modeling approach is presented, while the cost parameters used in the experiment are presented in Sect. "Cost parameters". Section "Results and discussion" discusses and compares the obtained results among comparative methods. Finally, conclusions and directions for future research are given in Sect. "Conclusions".

\section{Literature review}

Part of the planning process in Supply Chain Management (SCM) aims at finding the best possible supply chain configuration. These decisions are considered strategic because of their long time horizon and are tackled with facility location models. However, by considering certain aspects of the supply chain environment, these models can support the Supply Chain Network Design (SCND) phase (Melo et al. 2009). Moreover, dynamic facility location models, where the decisions are spread out over a longterm planning horizon and the decision variables are timedependent, are more compatible for tracking the dynamics of complex supply chains (Thanh et al. 2008; Babazadeh et al. 2012). The supply chain optimization in this study is perceived as determining which participants to include in the supply chain, their size and location, and establishing links among the participants. These problems are often very large and complex due to the number of plants, modes of transport, or relocation of warehouses and distribution centers, stochastic nature of demands, etc. Due to the complexities of a supply chain, very few analytical or mathematical models exist with the simplified problems limited by several assumptions (Ballou 2001; Beamon and Fernandes 2004; Cochran and Uribe 2005).

The optimization of supply chains often deals with the situation in which the interest is to find which of a large number of sets of model specifications lead to optimal output performance (April et al. 2003). Due to significant interactions between planning and scheduling decision levels, it is necessary to consider the simultaneous optimization of the planning and scheduling decisions to determine the global optimal solution. However, the complexity of the integrated problem causes computational load. The complexity has motivated the development of simulation tools for supply chain management that can capture the behavior of all the entities involved, their interactions, and the uncertainties associated with these systems. Simulation-based optimization is an emerging field which integrates optimization methods into simulation analysis. It is used for the evaluation of the objective value, and no further structural information is used. Therefore, a more detailed representation of complex supply chains is achieved, allowing the solution of larger optimization problems in acceptable time.

Most of today's simulators include possibilities to do a black-box parameter optimization of a simulation model. Glover et al. (1999) presented the successful development of OptQuest, an optimization tool box containing different algorithms (mainly metaheuristics) designed to optimize configuration decisions in simulation models. OptQuest uses iterative heuristics (Kleijnen 2008), and it can be used to utilize a combination of three meta-heuristics: Scatter Search (SS), Tabu Search (TS), and Neural Networks (NN) (Glover et al. 1999; Keskin et al. 2010; Abtahi and Bijari 2017). An example of successful application of scatter search within OptQuest is reported by Bulut (2001), to solve a multi-scenario optimization problem based on a 
large-scale linear programming problem. Cha-ume and Chiadamrong (2012) examined the relationship between inventory accuracy and financial performance using OptQuest to maximize the profit of the whole chain under various uncertainty conditions. Swisher et al. (2000) and Fu (2002) stated in their papers that there is still a big gap between optimization methods for simulation-based optimization used in commercial software and methods available in the research literature. Simulation-based optimization employs so-called metaheuristic methods, which do not guarantee an optimal solution and could provide poor results in the case of poor experimental settings.

The usefulness of simulation tools when combined with mathematical models has also been demonstrated with hybrid modeling approaches (Lee and Kim 2000). A hybrid analytical and simulation model is a mathematical model that combines identifiable analytical and simulation models. An analytical model is a set of equations that characterize a system or a problem entity, and a simulation model is a dynamic or an operating model of a system or problem entity that mimics the operating behavior of the system or problem entity. The analytical model tends to provide exact and static information, while the simulation model provides approximate and dynamic information about the system of interest or problem entity.

The earliest solution approach involving interactive use of simulation and optimization was developed by Nolan and Sovereign (1972). Their recursive approach involves an allocation of resources by a Linear Programming (LP) model at an aggregate level and a revision of productivity estimates by simulation of the schedules generated by optimization. Combining analytical and simulation modeling approaches can result in cost savings in solving complex problems (Sargent 1994). Over the years, hybrid simulation and analytical models have been applied to product and structure design (Kozan 1997; Mort and Tsai 1996), process design (Claver 1993), power plant performance prediction (Pereira et al. 1992), and in the semiconductor industry (Pierce 1994). However, a few hybrid analytical and simulation models have been used in production planning and production system design (Hsieh 2002). Kozan (1997) discussed the major factors influencing the transfer efficiency of seaport container terminals. An analytical model utilizing queueing techniques was constructed to draw inferences regarding strategies for a container terminal's service improvement. Later, the problem was formulated as a simulation model and a comparative analysis was performed on these models. Byrne and Bakir (1999) described the use of a hybrid simulation and analytical approach for production planning and demonstrated that their solution approach outperforms an LP approach alone. Their emphasis is on the use of an analytical model to generate an optimum production plan. Then, they used this plan as an input to a simulation model, which is subject to more realistic operational policies. If the simulation results are different from the analytical model, they are then used to adjust the constraints of the analytical models.

Byrne and Hossain (2005) developed an extended linear programming model for the hybrid modeling approach first proposed by Byrne and Bakir (1999). Their hybrid solution approach iteratively applies simulation and LP to solve a multi-period multi-product production planning problem. They obtained job workload and resource utilization through simulation and used LP to obtain the estimated optimal production plan that minimizes total costs. Ko et al. (2006) developed a hybrid optimization-simulation approach to design a distribution network for third party logistics (3PL) providers. They used a genetic algorithm to solve the optimization model that determines the distribution network structure. Subsequently, the solution model is applied to capture the uncertainty in client demand, orderpicking time, and travel time for the capacity plans of the warehouses. The simulation is used to estimate the average service time at each warehouse. Then, the service time is used to define appropriate throughput capacity constraints to be incorporated into subsequent optimization runs. If the simulation outputs satisfy the required performances, the procedure is terminated. Each of the iterative solution procedures described above is specific to a particular problem and exchange problem-specific parameters between simulation and optimization models.

The hybrid analytical and simulation approach in this study is used for giving a more realistic representation of the supply chain network. Using a hybrid modeling approach, we intend to determine the best storage locations and capacities of the warehouses, distribution centers, and retailers by incorporating the effect of uncertainty into the decision making process where an analytical model and a simulation-based optimization model are used iteratively. In a hybrid modeling framework, the analytical model is used to optimally determine the best storage locations among available choices under a certain set of parameters and constraints, and for given optimization criteria. These are imposed by the definition of the problem itself and/or the results of the simulation model. The simulation-based optimization model improves the decisions made by the analytical model further under a stochastic environment where the customer demand, delivery lead time, and plant production levels can fluctuate, giving the suitable sizes of warehouses, distribution centers, and retailers. The outline of the general framework for the hybrid modeling is given in Fig. 1.

The termination criteria control the mechanism between the analytical model and the simulation optimization 


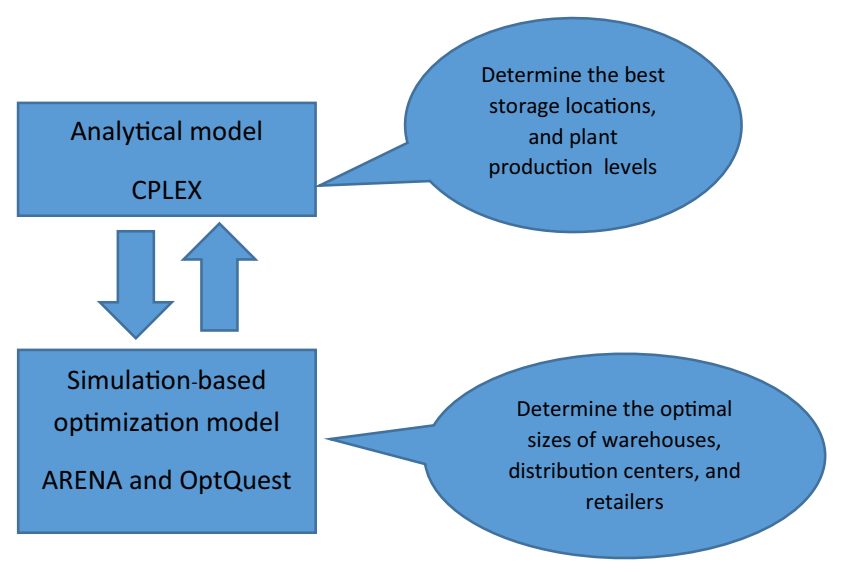

Fig. 1 Framework of hybrid modeling

model. This ensures termination when the hybrid approach's capability of considering the effect of uncertainties in making the decisions is satisfied or cannot be improved any further (by the revision/update process that modifies the problem parameters, constraints, and/or the objective function). Unlike other previous works, with additional optimization ability in the simulation model employed in this study, it is hoped that a faster solving time for finding convergence of the objective value can be achieved. We first discuss the details of the supply chain network problem and the structure of the SCN as a representation of the problem. Later, we present the hybrid modeling framework and discuss how it is employed to solve the problem.

\section{Supply chain network design}

The problem of Supply Chain Management (SCM) commonly arises in the following scenario: a number of retailers can be supplied from a number of distribution centers, which, in turn, are supplied from a number of warehouses. Then, each warehouse can be supplied from a number of plants. The challenge is to determine the number, location, and suitable size to use, so as to maximize the profit of the SCN. In this study, an agricultural product supply chain, such as rice, sugar, cassava, etc., is to be investigated. Once the production plants produce products, they cannot be kept at the plants to wait for the end customers to buy at the plants. Inventories need to be stored with the members in the chain. It is assumed that all members in the chain belong to one owner and share a common profit. Therefore, they can contribute to the same objective, which is to maximize the profit of the whole chain.

The origin for all activities in the supply chain is the predefined deterministic (but varying) demand of the end customers at the retailers. Using a periodic review every 3 months (quarterly), retailers pull the materials to replenish their taken inventory by the end customer from the distribution centers. In turn, the distribution centers pull the materials from the warehouses, and finally, the warehouses pull the materials from the plants. In this study, there are three production plants, four potential warehouses, four potential distribution centers, and four retailers, as shown in Fig. 2.

Warehouses and distribution centers (if established) have specified maximum capacities. Warehouses can be supplied from more than one production plant and can supply more than one distribution center. In the same manner, each retailer can be supplied from more than one distribution center. For each plant, the production level can also be subject to certain constraints.

Across the supply chain network, costs are created due to the establishment of warehouses, distribution centers, and retailers, due to production, storage, and transportation of materials from plants to warehouses, to distribution centers, and finally to retailers. The SCN decisions to be determined by the proposed model are as follows:

1. The production level of plants.

2. The storage locations in the network (to determine opening/closing of warehouses and distribution centers).

3. The capacity (to determine the size and construction cost) of warehouses, distribution centers, and retailers.

In the approach, first, we divide these SCN decisions into two groups. The first group of decisions is to be determined by the analytical model, and the second group is to be determined by the simulation-based optimization model. The optimal production level of plants and the best storage locations in the network can be determined by the analytical model, as they are not directly affected by uncertain factors, but are more influenced by the decisions suggested by the simulation-based optimization model, in which the stochastic data have been taken into consideration. The optimal capacities of warehouses, distribution centers, and retailers, which are directly affected by the stochastic data, especially fluctuating customer demand and delivery lead time, are determined by the simulationbased optimization model, which can incorporate uncertainty into the model. With the proposed hybrid analytical and simulation modeling approach, both models need to be identical and the system behavior over time is obtained by alternating between independent analytical and simulation models. The analytical model is a generalization of the simulation outcomes under deterministic data, and the simulation model is used to probe and identify the interrelationships and performance measures under stochastic data. 


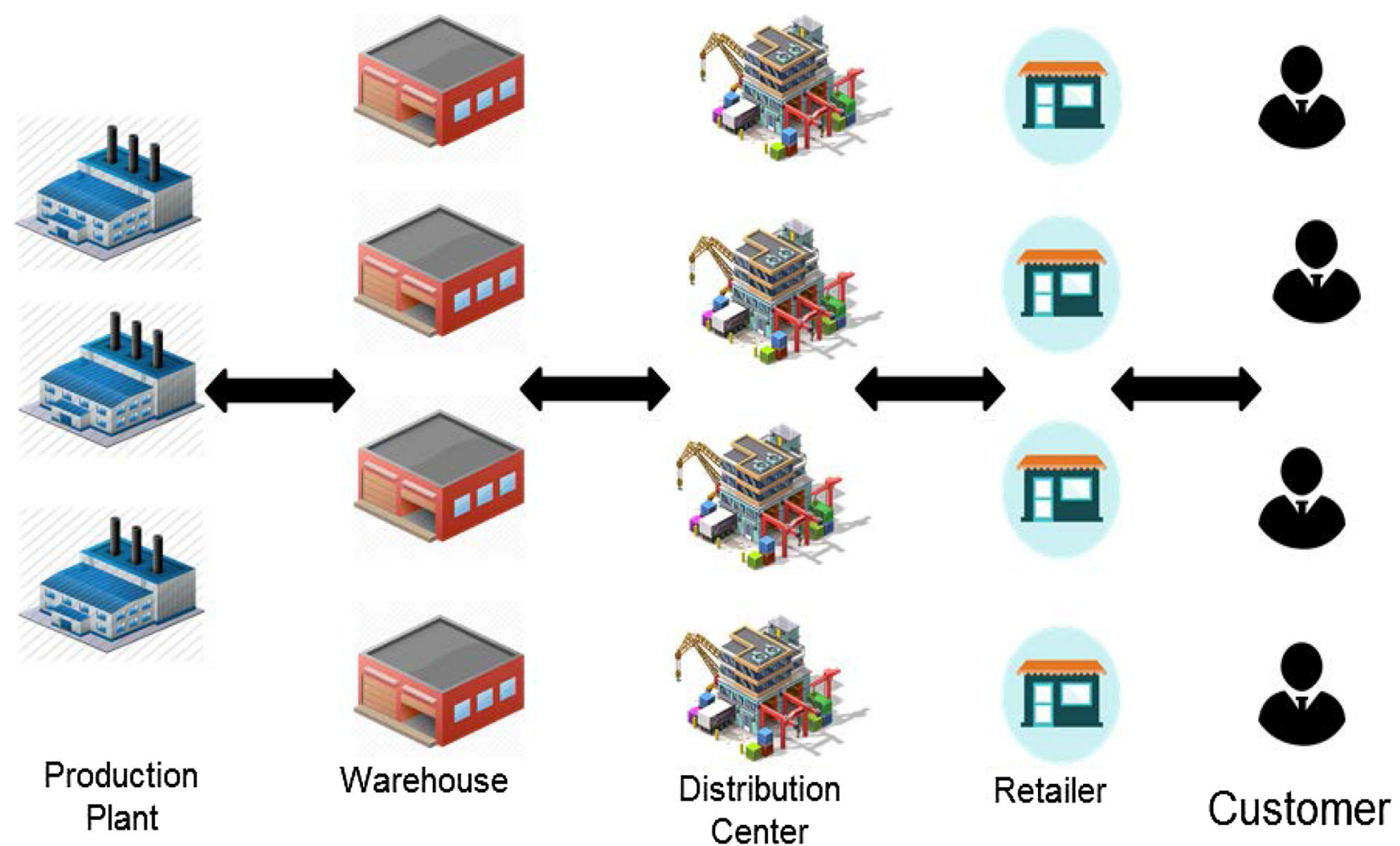

Fig. 2 Structure of the supply chain network

\section{Hybrid analytical-simulation modeling approach}

The idea in this study is to illustrate the hybrid approach for the solution of the SCN problem. The approach consists of building independent optimization models in both analytical and simulation models of the supply chain networks, as previously described, and integrating the solution strategy. The connection of the two models is shown in Fig. 3. In this work, we first construct the SCN analytical model with the given deterministic data in Mixed-Integer Linear Programming (MILP) using CPLEX. By setting the initial TSLs (maximum stock level) at each distribution center, warehouse, and retailer arbitrarily at 500 tonnes (Step 1), we obtain an optimal production level of each plant and a decision to open or close (deciding the material flow pattern) of certain warehouses and distribution centers to satisfy the given demand from all four retailers. In Step 2, the solution suggested by the analytical model is used to construct the simulation model, coded in ARENA, to test if the solution is viable with the stochastic data by checking the feasibility and the reliability of the solution, incorporating the effect of uncertainties into the model. In addition, OptQuest optimization tool embedded in ARENA is also employed to recommend the optimum TSL at each warehouse, distribution center, and retailer (as suggested by the analytical model). These levels of maximum kept inventory or the TSL at each member would be subject to uncertainty, which cannot be explained by the analytical model. It would be better and more realistic to obtain these results from the simulation-based optimization model, which suggests the best solution under an uncertain environment.

Then, the second iteration of the analytical model as seen in Step 3 starts again by inputting the TSLs obtained from the previous iteration of the simulation model (note that these levels are more likely TSLs under the applied uncertainties in the SCN) into the analytical model and solving it again to find the new optimal production levels of the plants and the new storage locations in the network (if different from the previous iteration). Having applied the uncertainties, the network system adjusts itself to contain more inventories and, hence, requires the production level to increase, to match the extra inventory. Taking the new production levels of the plants from the second iteration from the analytical model, the simulation-based optimization model is then run again to obtain a new solution of the TSLs of the warehouses, distribution centers, and retailers under uncertainties (Step 4).

At this time, the information gathered from the simulation-based optimization model is used to determine if the current solution, which is the profit of the supply chain network, satisfies the termination criteria in this 


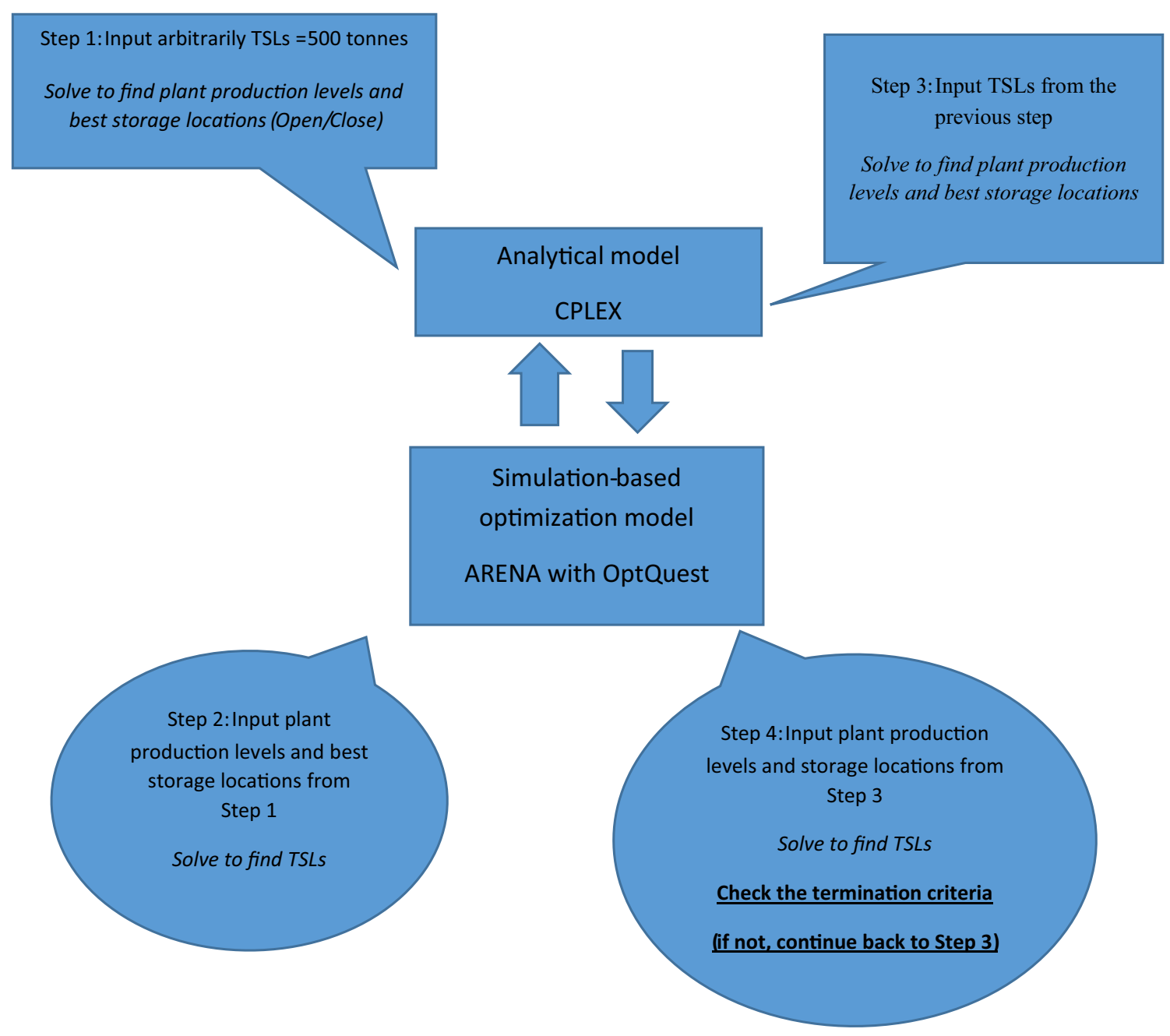

Fig. 3 Hybrid algorithm

study, which is set to be within a $5 \%$ difference from the optimal profit of the ideal case given by the analytical model. If the termination criteria are met, the solution suggested by the hybrid approach is accepted; otherwise, the results are used to revise the problem to be resolved by the analytical model in the third iteration, and so on. The revision of the problem may normally lead to changes in the parameter values, and/or addition and deletion of some constraints. Using the hybrid framework, a solution is found which honors the set of constraints described in the analytical model, along with an objective function to be minimized/maximized, meeting some performance criteria prescribed by the stochastic environment. The termination criteria control the mechanism between the analytical model and the simulation-based optimization model. This ensures termination when the hybrid approach's capability of considering the effect of uncertainties in making decisions is satisfied or cannot be improved any further.

\section{Analytical model}

A Mixed-Integer Linear Programming (MILP) is used to deal with the problem. The objective function is to maximize the profit of the whole supply chain network. The mathematical formulation and its notation can be presented as follows:

Indices:

$j=$ Plant

$m=$ Warehouse

$k=$ Distribution center

$l=$ Retailer

$t=$ Time period (quarters)

Parameters:

$\mathrm{PWDC}_{m, k}=$ Possible transportation route from warehouse $m$ to distribution center $k$

$\mathrm{PDCZ}_{k, l}=$ Possible transportation route from distribution center $k$ to retailer $l$ 
$\mathrm{PW}_{m}=$ Potential warehouse $m$

$\mathrm{PD}_{k}=$ Potential distribution center $k$

QPToWH $_{j, m, t}=$ Flow amount of materials transferred from plant $j$ to warehouse $m$ in time period $t$

$\mathrm{QWHToDC}_{m, k, t}=$ Flow amount of materials transferred from warehouse $m$ to distribution center $k$ in time period $t$ QDCToRT $_{k, l, t}=$ Flow amount of materials transferred from distribution center $k$ to retailer $l$ in time period $t$ $\mathrm{DM}_{l, t}=$ End customer demand for a product at retailer $l$ in time period $t$

$W_{m}=$ Size (Target stock level) of warehouse $m$

$D_{k}=$ Size (Target stock level) of distribution center $k$

$\mathrm{RT}_{l}=$ Size (Target stock level) of retailer $l$.

Cost parameters:

$\mathrm{CW}_{m}=$ Cost of establishing warehouse $m$ per tonne

$\mathrm{CD}_{k}=$ Cost of establishing distribution center $k$ per tonne

$\mathrm{CRT}_{l}=$ Cost of establishing retailer $l$ per tonne

Cprod $=$ Plant production cost per tonne

CplantToWH $\mathrm{W}_{j, m}=$ Transportation cost per tonne from plant $j$ to warehouse $m$

$\mathrm{CWHToDC}_{m, k}=$ Transportation cost per tonne from warehouse $m$ to distribution center $k$

$\mathrm{CDCToRT}_{k, l}=$ Transportation cost per tonne from distribution center $k$ to retailer $l$

$\mathrm{CIVP}_{j}=$ Cost per tonne of inventory holding of plant $j$ $\mathrm{CIVWH}_{m}=$ Cost per tonne of inventory holding of warehouse $m$

CIVDC $_{k}=$ Cost per tonne of inventory holding of distribution center $k$

$\mathrm{CIVRT}_{l}=$ Cost per tonne of inventory holding of retailer $l$

$\mathrm{DPR}=$ Depreciation rate per period

$\mathrm{FA}=$ Total fixed asset cost

$\mathrm{PC}_{t}=$ Production cost in time period $t$

$\mathrm{TC}_{t}=$ Transportation cost in time period $t$

$\mathrm{DP}_{t}=$ Depreciation cost in time period $t$

$\mathrm{NTS}_{t}=$ Net sales in time period $t$

PRICE $=$ Unit price per tonne

Profit $_{t}=$ Profit in time period $t$.

Decision variables:

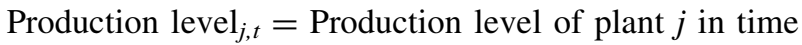
period $t$

$\operatorname{ILP}_{j, t}=$ Inventory level of plant $j$ at the end of time period $t$

$\mathrm{ILWH}_{m, t}=$ Inventory level of warehouse $m$ at the end of time period $t$

ILDC $_{k, t}=$ Inventory level of distribution center $k$ at the end of time period $t$

$\mathrm{ILRT}_{l, t}=$ Inventory level of retailer $l$ at the end of time period $t$.
Binary decision variables:

$\mathrm{PW}_{m}=1$ if warehouse $m$ is to be established, 0 otherwise

$\mathrm{PD}_{k}=1$ if distribution center $k$ is to be established, 0 otherwise.

\section{Network structure constraints}

The network structure constraints are for letting the supply chain send products to only the elements that exist, since there are four possible warehouses and four possible distribution centers. If the constraints are not created, materials would be sent to all the warehouses and distribution centers, even though they do not exist.

- Warehouse $m$ can only be established if there is a connection between warehouse $m$ itself and the distribution center $k$.

$\mathrm{PWDC}_{m, k} \leq \mathrm{PW}_{m} \quad \forall m, k$.

- If the distribution center does not exist, then the connection between the retailer $l$ and the distribution center does not exist.

$\mathrm{PDCZ}_{k, l} \leq \mathrm{PD}_{k} \quad \forall k, l$.

\section{Material balance constraints}

Inventory at each element in the supply chain is equal to the amount of product that flows into the elements, adding the amount of product that is left over from the previous time period before deducting the amount of product that flows out of the elements:

$$
\begin{gathered}
\mathrm{ILP}_{j, t}=\mathrm{ILP}_{j, t-1}+\left(\text { Production level }_{j, t}-\sum_{m} \mathrm{QPToWH}_{j, m, t}\right) \\
\forall j, m, t
\end{gathered}
$$

$$
\begin{aligned}
\mathrm{ILWH}_{m, t}= & \mathrm{ILWH}_{m, t-1} \\
& +\left(\sum_{j} \mathrm{QPToWH}_{j, m, t}-\sum_{k} \mathrm{QWHToDC}_{m, k, t}\right) \\
& \forall j, m, k, t \\
\mathrm{ILDC}_{k, t}= & \mathrm{ILDC}_{k, t-1} \\
+ & \left(\sum_{m} \mathrm{QWHToDC}_{m, k, t}-\sum_{l} \mathrm{QDCToCZ}_{k, l, t}\right) \\
& \forall m, k, l, t
\end{aligned}
$$




$$
\begin{aligned}
\operatorname{ILRT}_{l, t}= & \mathrm{ILRT}_{l, t-1} \\
& +\left(\sum_{m} \mathrm{QDCToRT}_{k, l, t}-\sum_{l} \mathrm{DM}_{l, t}\right) \quad \forall k, l, t
\end{aligned}
$$

where $\operatorname{ILP}_{j, 0}, \operatorname{ILWH}_{m, 0}, \operatorname{ILDC}_{k, 0}$, and $\operatorname{ILRT}_{l, 0}=0$.

\section{Production resource constraints}

The purpose of constructing the production resource constraints is to control the production level of plants, not to exceed the available resource capacity and not to be lower than zero.

Min production level $\leq$ Production level $_{j, t} \quad \forall j, t$

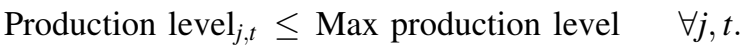

Minimum production level is set to zero and maximum production level is set to 10,000 tonnes per each time period (quarter).

\section{Capacity constraints for warehouses and distribution centers}

The capacity constraints for warehouses and distribution centers are constructed to determine the sizes of the warehouses and the distribution centers. The inventory level of a warehouse, a distribution center, and a retailer is less than or equal to the capacity of the warehouses, distribution centers, and retailers, respectively.

$$
\begin{aligned}
& \mathrm{ILWH}_{m, t} \leq W_{m} \times \mathrm{PW}_{m} \quad \forall m, t \\
& \mathrm{ILDC}_{k, t} \leq D_{k} \times \mathrm{PD}_{k} \quad \forall k, t \\
& \mathrm{ILRT}_{l, t} \leq \mathrm{RT}_{l} \quad \forall l, t .
\end{aligned}
$$

\section{Financial operation constraints}

The financial operation constraints deal with the profit and cost calculation throughout the supply chain network. These costs include the plant production cost (PC), transportation cost (TC), inventory holding cost (HC), and building depreciation cost (DP), where the profit is the revenue (NTS) minus these costs. As addressed by Rabbani et al. (2016), there are plentiful papers in the field of supply chain network design, but there are no papers which regard the depreciation cost as an important element of the model. The depreciation cost of the fixed asset is a crucial element for designing a supply chain network:

$$
\begin{aligned}
\mathrm{PC}_{t}= & \sum_{j} \mathrm{CProd}_{j} \times \text { Production level }_{j, t} \quad \forall j, t \\
\mathrm{TC}_{t}= & \sum_{j, m} \text { CplantToWH }_{j, m} \times \mathrm{QPToWH}_{j, m, t} \\
& +\sum_{m, k} \mathrm{CWHToDC}_{m, k} \times \mathrm{QWHToDC}_{m, k, t} \\
& +\sum_{k, l} \mathrm{CDCToCZ}_{k, l} \times \mathrm{QDCToCZ}_{k, l, t} \quad \forall j, m, k, l, t
\end{aligned}
$$

$$
\begin{aligned}
\mathrm{HC}_{t}= & \sum_{j} \mathrm{CIVP}_{l} \times\left(\left(\mathrm{ILP}_{j, t}+\mathrm{ILP}_{j, t-1}\right) / 2\right) \\
& +\sum_{m} \mathrm{CIVWH}_{m} \times\left(\left(\mathrm{ILWH}_{m, t}+\mathrm{ILWH}_{m, t-1}\right) / 2\right) \\
& +\sum_{k} \mathrm{CIVDC}_{k} \times\left(\left(\mathrm{ILDC}_{k, t}+\mathrm{ILDC}_{k, t-1}\right) / 2\right) \\
& +\sum_{l} \mathrm{CIVRT}_{l} \times\left(\left(\mathrm{ILRT}_{l, t}+\mathrm{ILRT}_{l, t-1}\right) / 2\right) \\
& \forall j, m, k, l, t
\end{aligned}
$$

$$
\begin{aligned}
\mathrm{FA}= & \sum_{m} W_{m} \times \mathrm{CW}_{m} \times \mathrm{PW}_{m}+\sum_{k} D_{k} \times \mathrm{CD}_{k} \\
& \times \mathrm{PD}_{k}+\sum_{l} \mathrm{RT}_{l} \times \mathrm{CRT}_{l} \quad \forall m, k, l, t
\end{aligned}
$$

$\mathrm{DP}_{t}=\mathrm{DPR}_{t} \times \mathrm{FA}_{t} \quad \forall t$

$\mathrm{NTS}_{t}=\mathrm{PRICE} \times \sum_{l} \mathrm{DM}_{l, t} \quad \forall l, t$

Profit $_{t}=\mathrm{NTS}_{t}-\mathrm{PC}_{t}-\mathrm{TC}_{t}-\mathrm{HC}_{t}-\mathrm{DP}_{t} \quad \forall t$.

\section{Simulation-based optimization model}

This model is used to generate products, store them in several places in the SCN, and deliver them if there is demand. Without uncertainty in the model, the simulation model yields an identical solution to the analytical model, as they are required to exchange certain values later on. In this study, simulation and optimization procedures are completed using ARENA commercial software and the OptQuest optimization tool. There are three groups of main decision variables in the model. The first group is TSLs at 
Table 1 Level of the mean values of uncertainty in the simulation model

\begin{tabular}{lllll}
\hline & Quarter 1 & Quarter 2 & Quarter 3 & Quarter 4 \\
\hline End customer demand at retailer 1 (tonnes) & 654 & 745 & 456 & 398 \\
End customer demand at retailer 2 (tonnes) & 986 & 678 & 598 & 698 \\
End customer demand at retailer 3 (tonnes) & 543 & 879 & 578 & 777 \\
End customer demand at retailer 4 (tonnes) & 551 & 657 & 890 & 654 \\
Delivery lead time (minutes) & 240 min & As obtained by the solution of the analytical model from the previous iteration. \\
Plant production level (tonnes) &
\end{tabular}

Remarks: three levels of uncertainty are experimented by varying the mean values of the end customer demand, delivery lead time, and plant production level simultaneously by 1,5 , and $10 \%$ from the mean values. All values are normally distributed

each warehouse (WH1, WH2, WH3, and WH4), the second group is the TSLs at each distribution center (DC1, DC2, DC3, and DC4), and the last group is the TSLs at each retailer (RL1, RL2, RL3, and RL4). The optimal settings for these decision variables are searched for by OptQuest. The lower and upper bounds of the searching boundary in each variable are guaranteed to be large enough to ensure that the optimal settings are inside the searching boundary.

Since the simulation model may contain stochastic and nonlinear elements, it is necessary to perform several simulation runs and combine the results. Ten replications are simulated with 1 year (4 quarters) per replication. As a result, the presented optimal profits of the chain are the average of best values from 10 replications. To measure the quality of our solutions in a stochastic environment, we prepare a set of test examples, including three factors of uncertainty (customer demand, delivery lead time, and plant production level), as well as each instance with 3 levels of uncertainty $(1,5$, and $10 \%$ deviation from the mean values), as these uncertainties are estimated to follow the normal distribution. Table 1 shows the mean values of the end customer demand at the retailers, delivery lead times, and plant production levels.

\section{Cost parameters}

The objective of the study is to maximize the profit of the $\mathrm{SCN}$. There are four major costs affected by supply chain decisions, i.e., production cost at the plants, transportation cost, holding cost, and building depreciation costs of warehouses, distribution centers, and retailers in the network, while the revenue is generated at the most downstream members, which are the retailers. It is also assumed that shortage is not allowed, so all members need to keep a sufficient amount of inventory at all times. Please note that all costs are based on Thai Baht. Currently, 1USD is around 35 Baht.
Table 2 Transportation cost from plants to warehouses (Baht/tonne)

\begin{tabular}{rrrrr}
\hline & WH1 & WH2 & WH3 & WH4 \\
\hline P1 & 200 & 1250 & 1180 & 1550 \\
P2 & 1370 & 2000 & 150 & 1300 \\
P3 & 2540 & 1700 & 1640 & 250 \\
\hline
\end{tabular}

Table 3 Transportation cost from warehouse to distribution centers (Baht/tonne)

\begin{tabular}{lrrrr}
\hline & DC1 & DC2 & DC3 & DC4 \\
\hline WH1 & 240 & 3670 & 1650 & 2125 \\
WH2 & 1500 & 1855 & 970 & 1630 \\
WH3 & 1300 & 1540 & 200 & 3420 \\
WH4 & 2590 & 3260 & 3980 & 225 \\
\hline
\end{tabular}

The selling price is set at 20,000 Baht per tonne, while the production cost is at 3,000 Baht per tonne, and the holding cost is estimated at $40 \%$ per year, which is around 100 Baht per tonne per quarter. As the sizes of warehouses, distribution centers, and retailers play an important part in the profit calculation and determining their depreciation costs, we have set the building construction cost to keep a tonne of product of each member in the chain at 500 Baht. As a result, when one member needs to keep a certain level of inventory (determined by its TSL, as it represents the maximum level of kept inventory), the cost of the building construction can be calculated, and hence, the value of its depreciation cost per year, which is $10 \%$ per year, can be generated. The transportation cost depends on the distance between a pair of locations per tonne of delivery. We measure these distances among members in the SCN and estimate the transportation cost among them, in which the cost per tonne between each pair is presented in Tables 2, 3 and 4. 
Table 4 Transportation cost from distribution centers to retailers (Baht/tonne)

\begin{tabular}{lrrrr}
\hline & RL1 & RL2 & RL3 & RL4 \\
\hline DC1 & 120 & 325 & 4025 & 3425 \\
DC2 & 1500 & 2420 & 3550 & 2440 \\
DC3 & 3150 & 2770 & 220 & 4360 \\
DC4 & 2410 & 3070 & 3050 & 320 \\
\hline
\end{tabular}

\section{Results and discussion}

We investigate the advantages of the hybrid analytical and simulation modeling approach, as compared to the traditional individual optimization methods with empirical tests, using a set of 3 methods, i.e., 1. Analytical model, 2. Simulation-based optimization model with OptQuest, and 3. Hybrid analytical and simulation modeling approach. To achieve this goal, all methods need to operate under the same conditions, so the comparison can be fairly made. Results of each method are analyzed and presented as follows:

\section{Analytical model (by CPLEX)}

This is an ideal case when there is no uncertainty in the model. The obtained solution gives the highest profit of the supply chain network and is used as the base case of the decisions to be made in each instance. Without uncertainty and assuming no material delivery lead time, the model recommends keeping no inventory at all members (TSLs of warehouses, distribution centers, and retailers are zero). As a result, all members receive all requested inventories at the time they are requested, and hence, keeping an inventory would not be required. The material delivery time is assumed to be zero, to simplify the model formulation and reduce the number of solving periods; otherwise, the solving period must be sub-divided into a number of small periods for the shorter lead time period. In addition, for a fair comparison with other models and presenting more meaningful results, we set the TSLs (to determine the size of each member) equal to the ones obtained from the hybrid analytical and simulation modeling approach, and let the analytical model recommend only the plant production levels and the storage locations in the supply chain network. Tables 5, 6 show the optimal production levels and the storage locations as determined by the analytical model, respectively. Table 7 presents the obtained profits from all three levels of uncertainty.

The result from the analytical model suggests to open warehouses no. 1, 3, and 4 and distribution centers no. 1, 3, and 4 (see Table 5), as well as recommends that each plant produces the amount, as shown in Table 6. It is not surprising that the plants need to produce more at the beginning during the first quarter, since they are forced to produce more to fill the TSLs of opening members, as they have no inventory at the beginning. After the first quarter, the plants produce only to replenish the inventory taken from the downstream members. As there is no uncertainty in the model, the amount to produce of all plants for each quarter is similar, despite the different levels of uncertainty
Table 5 Optimal values of decision variables for warehouses, distribution centers, and retailers from the analytical model

Table 6 Optimal values of decision variables (production level) for plants from the analytical model

\begin{tabular}{|c|c|c|c|c|c|c|c|c|}
\hline \multirow[t]{2}{*}{ Decision variables } & \multicolumn{4}{|c|}{ Warehouses } & \multicolumn{4}{|c|}{ Distribution centers } \\
\hline & WH1 & WH2 & WH3 & WH4 & DC1 & $\mathrm{DC} 2$ & DC3 & DC4 \\
\hline Open/close & 1 & 0 & 1 & 1 & 1 & 0 & 1 & 1 \\
\hline \multicolumn{9}{|l|}{ Open $=1$} \\
\hline Close $=0$ & & & & & & & & \\
\hline
\end{tabular}

\begin{tabular}{llllll}
\hline Plant & Level of uncertainty & Period & & \\
\cline { 3 - 5 } & & Quarter 1 & Quarter 2 & Quarter 3 & Quarter 4 \\
\hline P1 & $1 \%$ & 8340 & 1423 & 1054 & 1096 \\
P2 & & 4843 & 879 & 578 & 777 \\
P3 & & 3751 & 657 & 890 & 654 \\
P1 & $5 \%$ & 8240 & 1423 & 1054 & 1096 \\
P2 & & 3643 & 879 & 578 & 777 \\
P3 & & 4151 & 657 & 890 & 654 \\
P1 & $10 \%$ & 9640 & 1423 & 1054 & 1096 \\
P2 & & 4943 & 879 & 578 & 777 \\
P3 & & 4451 & 657 & 890 & 654 \\
\hline
\end{tabular}


Table 7 Profits obtained from the analytical model using CPLEX

\begin{tabular}{ll}
\hline Level of uncertainty & Profit (Baht) \\
\hline $1 \%$ & $176,720,000$ \\
$5 \%$ & $175,090,000$ \\
$10 \%$ & $170,490,000$ \\
\hline
\end{tabular}

(except the first quarter as explained above). It is also found that the profit of the chain decreases when the level of uncertainty increases (see Table 7), as higher expenses are incurred to produce more products and keep a higher level of inventory, while the revenue is stable, as the same amount of end customer demand is used in all cases.

\section{Simulation-based optimization model}

The OptQuest optimization tool embedded in ARENA is used to search for the optimal SCN decisions under 3 levels of uncertainty. Suggested outcomes from OptQuest recommend to open all warehouses and distribution centers. Tables 8,9 show the optimal values of decision variables for plants, warehouses, distribution centers, and retailers suggested by OptQuest. Table 10 shows the obtained profits. Please note that the production levels of the plants and the TSLs of downstream members are searched to find
Table 10 Profits obtained from the simulation-based optimization model with OptQuest

\begin{tabular}{lll}
\hline $\begin{array}{l}\text { Level of } \\
\text { uncertainty }\end{array}$ & $\begin{array}{l}\text { Profit } \\
\text { (Baht) }\end{array}$ & $\begin{array}{l}\text { Percentage difference between the } \\
\text { simulation-based optimization model } \\
\text { and the analytical model }\end{array}$ \\
\hline $1 \%$ & $152,792,400$ & $-13.54 \%$ \\
$5 \%$ & $151,609,100$ & $-13.41 \%$ \\
$10 \%$ & $136,819,200$ & $-19.75 \%$ \\
\hline
\end{tabular}

their optimal levels at a step size of 100 tonnes to reduce the solving time of OptQuest.

When the level of uncertainty increases, the system generally adjusts itself by increasing the level of kept inventory (higher level of TSLs) and, hence, a lower level of profit in the chain. It is observed that the profits obtained from the simulation-based optimization model with OptQuest, where uncertainty exists, are far lower than the ones from the analytical model by CPLEX, which are run under no uncertainty conditions, as shown in Table 10. Furthermore, it is noticed that the obtained profit decreases considerably when the level of uncertainty increases beyond $10 \%$.

Table 8 Optimal values of decision variables for warehouses, distribution centers, and retailers from the simulation-based optimization model

\begin{tabular}{|c|c|c|c|c|c|c|c|c|c|c|c|c|c|}
\hline \multirow[t]{2}{*}{ Level of uncertainty } & \multirow[t]{2}{*}{ Decision variables } & \multicolumn{4}{|c|}{ Warehouses } & \multicolumn{4}{|c|}{ Distribution centers } & \multicolumn{4}{|c|}{ Retailers } \\
\hline & & WH1 & WH2 & WH3 & WH4 & DC1 & $\mathrm{DC} 2$ & DC3 & DC4 & RL1 & RL2 & RL3 & RL4 \\
\hline All cases $(1 \%, 5 \%, 10 \%)$ & $\begin{array}{l}\text { Open/Close } \\
\text { Open }=1 \\
\text { Close }=0\end{array}$ & 1 & 1 & 1 & 1 & 1 & 1 & 1 & 1 & - & - & - & - \\
\hline $1 \%$ & TSL (tonnes) & 4700 & 2800 & 2600 & 3600 & 1600 & 1500 & 1200 & 900 & 500 & 1100 & 1000 & 1800 \\
\hline $5 \%$ & TSL (tonnes) & 1700 & 400 & 2400 & 2900 & 2000 & 1200 & 2200 & 1400 & 600 & 1200 & 1300 & 2000 \\
\hline $10 \%$ & TSL (tonnes) & 2900 & 700 & 4100 & 1500 & 1900 & 1300 & 1000 & 2100 & 800 & 1700 & 1100 & 1500 \\
\hline
\end{tabular}

Table 9 Optimal values of decision variables (production level) for plants from the simulation-based optimization model

\begin{tabular}{llllll}
\hline Plant & Level of uncertainty & Period & & \\
\cline { 3 - 6 } & & Quarter 1 (tonnes) & Quarter 2 (tonnes) & Quarter 3 (tonnes) & Quarter 4 (tonnes) \\
\hline P1 & $1 \%$ & 5700 & 1600 & 1000 & 1900 \\
P2 & & 5800 & 1000 & 1200 & 900 \\
P3 & & 4700 & 1000 & 1300 & 1900 \\
P1 & $5 \%$ & 6300 & 1100 & 800 & 1400 \\
P2 & & 4500 & 1000 & 1100 & 1400 \\
P3 & & 5600 & 1300 & 1700 & 900 \\
P1 & $10 \%$ & 8100 & 1400 & 1000 & 800 \\
P2 & & 8600 & 900 & 1200 & 1200 \\
P3 & & 3600 & 900 & 800 & 1100 \\
\hline
\end{tabular}


Table 11 Optimal values of decision variables (production level) for plants from the hybrid approach

\begin{tabular}{llllll}
\hline Plant & Level of uncertainty & Period & & \\
\cline { 3 - 6 } & & Quarter 1 (tonnes) & Quarter 2 (tonnes) & Quarter 3 (tonnes) & Quarter 4 (tonnes) \\
\hline P1 & $1 \%$ & 8445 & 1432 & 1048 & 1093 \\
P2 & & 5838 & 884 & 577 & 787 \\
P3 & & 4458 & 655 & 879 & 647 \\
P1 & $5 \%$ & 3640 & 1423 & 1054 & 1096 \\
P2 & & 2043 & 879 & 578 & 777 \\
P3 & & 2051 & 657 & 890 & 654 \\
P1 & $10 \%$ & 8127 & 1427 & 1078 & 1118 \\
P2 & & 3406 & 850 & 555 & 774 \\
P3 & & 3698 & 632 & 953 & 626 \\
\hline
\end{tabular}

Table 12 Optimal values of decision variables for warehouses, distribution centers, and retailers from the hybrid approach

\begin{tabular}{|c|c|c|c|c|c|c|c|c|c|c|c|c|c|}
\hline \multirow[t]{2}{*}{ Level of uncertainty } & \multirow[t]{2}{*}{ Decision variables } & \multicolumn{4}{|c|}{ Warehouses } & \multicolumn{4}{|c|}{ Distribution centers } & \multicolumn{4}{|c|}{ Retailers } \\
\hline & & WH1 & WH2 & WH3 & WH4 & $\mathrm{DC} 1$ & $\mathrm{DC} 2$ & DC3 & $\mathrm{DC} 4$ & RL1 & RL2 & RL3 & RL4 \\
\hline All cases & Open/Close & 1 & 0 & 1 & 1 & 1 & 0 & 1 & 1 & - & - & - & - \\
\hline$(1 \%, 5 \%, 10 \%)$ & $\begin{array}{l}\text { Open }=1 \\
\text { Close }=0\end{array}$ & & & & & & & & & & & & \\
\hline $1 \%$ & TSL (tonnes) & 2300 & 0 & 1900 & 1400 & 2200 & 0 & 900 & 900 & 800 & 1400 & 1500 & 900 \\
\hline $5 \%$ & TSL (tonnes) & 2200 & 0 & 800 & 1300 & 2300 & 0 & 1200 & 1300 & 1000 & 1100 & 1100 & 1000 \\
\hline $10 \%$ & TSL (tonnes) & 3400 & 0 & 1500 & 1300 & 2400 & 0 & 1700 & 1500 & 1200 & 1700 & 1200 & 1100 \\
\hline
\end{tabular}

\section{Hybrid analytical and simulation modeling approach}

The hybrid approach uses the advantages of both modeling methods while avoiding the problems of both methods. From Tables 11 and 12, it is observed that the best storage locations suggested by the hybrid approach are similar to the solution given by the analytical model, since it adopts the best storage locations from the analytical model, while the production levels at the plants and the sizes of warehouses, distribution centers, and retailers, which are optimized separately, are somewhat different. However, when the level of uncertainty increases, these sizes increase accordingly, as explained previously.

The resulting optimal SCN design, through iterations for each period, is given in Table 13. The result shows maximum stopping iterations of three iterations in the case of $1 \%$ uncertainty, while only two iterations in the case of a higher level of uncertainty. With this stopping criteria, there is less than $2 \%$ difference in the profit of the chain from the optimal values given by the analytical model, although the stopping criteria is set to be within 5\%. This is considered to be quite good as compared to the optimum under a deterministic environment. Although it is not possible to prove general convergence for all our test instances, we observe fast convergence of the objective values for the proposed hybrid approach. Incorporating optimization in the simulation algorithm decreases the solving time and brings faster convergence of the objective values.

Even though we did not precisely record the solving time of these three comparative methods, it was found that the analytical model solved by CPLEX alone takes a matter of minutes to solve each instance. The simulation-based optimization model with OptQuest takes a long time (longer than $20 \mathrm{~h}$ for each instance) to yield the optimal value of profit, recommending the optimal design of the supply chain network. The hybrid analytical and simulation modeling approach takes somewhere in between CPLEX and OptQuest. All instances were recorded to take less than half of the solving time of the traditional simulation-based optimization model.

\section{Conclusions}

In this paper, we present a hybrid analytical and simulation optimization approach for solving a design problem in a supply chain network. The study presents and examines a general solution methodology that obtains an estimated global optimum for combinatorial optimization problems 
Table 13 Profits and number of iterations to reach the stopping criteria from the hybrid approach

\begin{tabular}{lllll}
\hline $\begin{array}{l}\text { Level of } \\
\text { uncertainty }\end{array}$ & $\begin{array}{l}\text { Profit } \\
\text { (Baht) }\end{array}$ & $\begin{array}{l}\text { Number of } \\
\text { iterations }\end{array}$ & $\begin{array}{l}\text { Percentage difference between the hybrid } \\
\text { approach and the analytical model }\end{array}$ & $\begin{array}{l}\text { Percentage difference between the hybrid approach } \\
\text { and the simulation-based optimization model }\end{array}$ \\
\hline $1 \%$ & $175,063,400$ & 3 & $-0.95 \% \sqrt{2}$ & $+14.58 \%$ \\
$5 \%$ & $171,808,300$ & 2 & $-1.87 \%$ & $+13.32 \%$ \\
$10 \%$ & $169,242,300$ & 2 & $-0.73 \%$ & $+23.70 \%$ \\
\hline
\end{tabular}

with components of uncertainty. The approach is initialized by solving the analytical model to optimality with the given deterministic data, which is usually prescribed by the expected value of the uncertain problem input. The solution, suggested by the analytical model, is used to construct the simulation-based optimization model, to test if the solution is viable with the stochastic data by checking the feasibility and the reliability of the solution, incorporating the effect of uncertainty and further optimized parameters, which are subject to an uncertain environment. The information gathered from the simulation-based optimization model is used to determine if the current solution satisfies the termination criteria. If the termination criteria are met, the solution suggested by the hybrid approach is accepted; otherwise, the results are used to revise the problem, to be resolved by the analytical model. The revision of the problem may lead to changes in the parameter values or constraints. Using the hybrid framework, a solution is found, which honors the set of constraints described in the analytical model, along with an objective function to be minimized/maximized. This meets performance criteria prescribed by the stochastic environment with closer to optimal results and shorter solving time than traditional simulation-based optimization modeling methods.

The primary findings reported in this paper are promising; however, there are some open research issues that remain to be examined. This study only examines the use of the hybrid approach with the SCN design. Since our approach is generalized and can be applied to solve different types of combinatorial problems, it would be interesting to consider different problems with various components of uncertainty and differing cost structures in future research. As future work, an improvement of model formulation and the generalization of the representation of the problem could still be further explored. Multiple pickup locations with partial pick-up loads and allowing shortages or back ordering can be included in the case study. The treatment of uncertainty should be further explored. Furthermore, multi-objective approaches can also be addressed as an extension of the present study.

Open Access This article is distributed under the terms of the Creative Commons Attribution 4.0 International License (http://crea tivecommons.org/licenses/by/4.0/), which permits unrestricted use, distribution, and reproduction in any medium, provided you give appropriate credit to the original author(s) and the source, provide a link to the Creative Commons license, and indicate if changes were made.

\section{References}

Abtahi AR, Bijari A (2017) A noval hybrid meta-heuristic technique applied to the well-known benchmark optimization problems. J Ind Eng Int 13:93-105. doi:10.1007/s40092-016-0170-x

April J, Glover F, Kelly J, Laguna M (2003) Practical introduction to simulation optimization. In: The 2003 winter simulation conference, New Orleans, LA, pp 71-78

Babazadeh R, Razmi J, Ghodsi R (2012) Supply chain network design problem for a new market opportunity in an agile manufacturing system. J Ind Eng Int 8:19

Ballou RH (2001) Unresolved issues in supply chain network design. Inform Syst Front 3(4):417-426. doi:10.1023/A:1012872704057

Banks J, Carson JS, Nicol BL, Nelson DM (2001) Discrete event system simulation. Prentice Hall, Upper Saddle River

Beamon BM, Fernandes C (2004) Supply chain network configuration for product recovery. Prod Plan Control 15:270-281

Bulut G (2001) Robust multi-scenario optimization of an air expeditionary force structure applying scatter search to the combat forces assessment model. MS Thesis, Air Force Institute of Technology, Ohio, USA

Byrne MD, Bakir MA (1999) Production planning using a hybrid simulation-analytical approach. Int J Prod Econ 59(1):305-311

Byrne MD, Hossain MM (2005) Production planning: an improved hybrid approach. Int J Prod Econ 93-94:225-229

Chandra C, Grabis J (2007) Supply chain configuration: concept, solutions, and applications. Springer, New York

Cha-ume K, Chiadamrong N (2012) Simulation of retail supply chain behaviour and financial impact in an uncertain environment. Int $\mathrm{J}$ Logist Syst Manag 13(2):162-186

Claver JF, Coste B (1993) Paste plant design and control: a new approach. In: Light metals 1993 proceedings of the 122nd annual meeting, Denver, Colorado, pp 641-645

Cochran JK, Uribe AM (2005) A set covering formulation for agile capacity planning within supply chains. Int $\mathrm{J}$ Prod Econ 95:139-149

Fu MC (2002) Optimization for simulation: theory vs practice. Inform J Comput 14:192-215

Glover F, Kelly JP, Laguna M (1999) New advances for wedding optimization and simulation. In: The 1999 Winter Simulation Conference, pp 255-260

Hsieh SJ (2002) Hybrid analytic and simulation models for assembly line design and production planning. Simul Pract Theory 10:87-108

Izadi A, Kimiagari AM (2014) Distribution network design under demand uncertainty using genetic algorithm and Monte Carlo simulation approach: a case study in pharmaceutical industry. J Ind Eng Int 10:50. doi:10.1007/s40092-014-0050-1 
Keskin B, Melouk S, Meuer I (2010) A simulation-optimization approach for integrated sourcing and inventory decisions. Int $\mathrm{J}$ Comput Oper Res 37(9):1648-1661

Kleijnen JPC (2008) Design and analysis of simulation experiments. Springer, New York. doi:10.1007/978-0-387-71813-2

Ko HJ, Ko CS, Kim T (2006) A hybrid optimization/simulation approach for a distribution network design of 3PLs. Comput Ind Eng 50(4):440-449

Kozan E (1997) Comparison of analytical and simulation planning models of seaport container terminal. Transport Plan Techn 20(3):235-248

Lee YH, Kim SH (2000) Optimal production-distribution planning in supply chain management using a hybrid simulation-analytic approach. In: The 2000 winter simulation conference, Orlando, FL, pp 1252-1259

Mehrdad M, Tu N, Miao L (2015) A hybrid solution approach for a multi-objective closed-loop logistics network under uncertainty. J Ind Eng Int 11:237-252. doi:10.1007/s40092-014-0089-Z

Melo MT, Nickel S, Saldanha-da-Gama F (2009) Facility location and supply chain management-a review. Eur J Oper Res 196(2):401-412

Mort N, Tsai CS (1996) Simulation and optimization in manufacturing systems using Taguchi methods. In: UKACC international conference on control' 96, University of Exeter, pp 467-471

Nikolopoulou A, Ierapetritou MG (2012) Hybrid simulation based optimization approach for supply chain management. Comput Chem Eng 47(20): 183-193

Nolan RL, Sovereign MG (1972) A recursive optimization and simulation approach to analysis with an application to transportation systems. Manag Sci 18(12):676-690
Pereira MVF, Maceira MEP, Oliveria GC, Pinto LMVG (1992) Combining analytical models and Monte Carlo techniques in probabilistic power system analysis. IEEE $\mathrm{T}$ Power Syst $7(1): 265-272$

Pierce NG (1994) Golden nuggets of AMHS modeling and design for semiconductor wafer fabrication. In: The IEEE/SEMI 1994 advanced semiconductor manufacturing conference and workshop, Cambridge, MA, pp 200-204

Rabbani M, Ramezankhani F, Giahi R, Farshbaf-Geranmayeh A (2016) Biofuel supply chain considering depreciation cost of installed plants. J Ind Eng Int 12:221-235. doi:10.1007/s40092015-0139-1

Sargent RG (1994) Historical view of hybrid simulation/analytic models. In: The 1994 winter simulation conference. FL, pp 383-386

Swisher JR, Jacobson SH, Hyden PD, Schruben LW (2000) A survey of simulation and optimization techniques and procedures. In: The 2000 winter simulation conference, FL, pp 119-128

Tabrizi BH, Razmi J (2013) A multi-period distribution network design model under demand uncertainty. J Ind Eng Int. doi:10. 1186/2251-712X-9-13

Tavakkoli-Moghaddam R, Forouzanfar F, Ebrahimnejad S (2013) Incorporating location, routing, and inventory decisions in a biobjective supply chain design problem with risk-pooling. J Ind Eng Int 9:19

Thanh PN, Bostel N, Peton O (2008) A dynamic model for facility location in the design of complex supply chains. Int J Prod Econ 113(2):678-693 